\title{
Painful swelling of the knee and ankle: presentation
}

\author{
Rakesh Mohankumar • Haren Varia • Lipsita Patnaik • \\ Shabbir Susnerwala
}

Received: 10 October 2010 /Revised: 31 January 2011 / Accepted: 3 February 2011 /Published online: 25 February 2011

(C) ISS 2011

\section{History}

A 66-year-old woman presented with an 18-month history of discomfort and swelling of her right knee and right ankle. She also noticed some skin lesions adjacent to her knee swelling. No history of previous trauma was identified (Figs. 1, 2, 3 and 4).
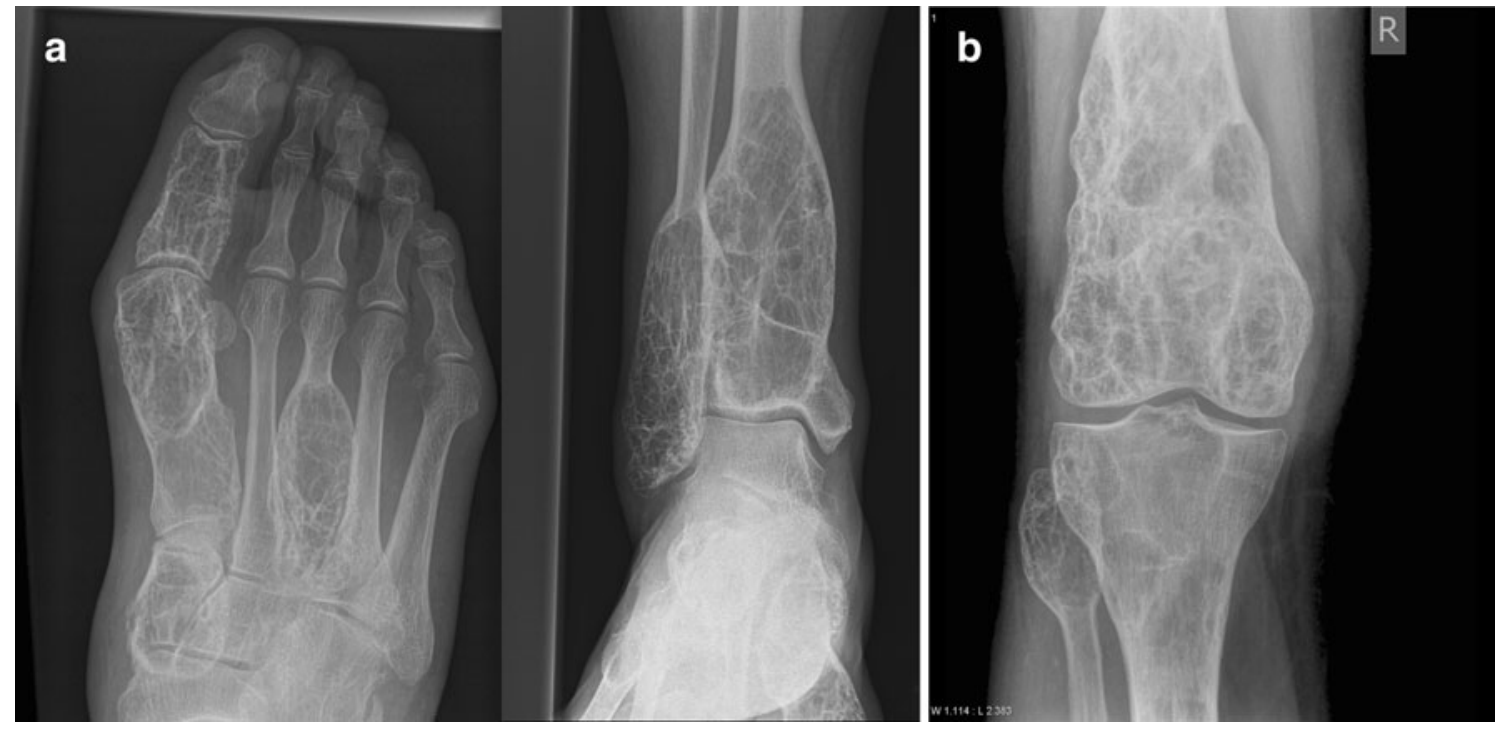

Fig. 1 a AP radiographs of the right ankle and foot. b AP radiograph of the right knee

The diagnosis can be found at doi:10.1007/s00256-011-1126-2.

R. Mohankumar $\cdot$ H. Varia

Department of Radiology, Blackpool Victoria Hospital,

Whinney Heys Road, Blackpool,

Lancashire FY3 8NR, United Kingdom

L. Patnaik

Department of Histopathology, Blackpool Victoria Hospital,

Whinney Heys Road, Blackpool, Lancashire FY3 8NR, UK

\section{S. Susnerwala}

Department of Clinical Oncology, Blackpool Victoria Hospital, Whinney Heys Road, Blackpool,

Lancashire FY3 8NR, UK

R. Mohankumar $(\bowtie)$

3, Ladybridge Avenue, Worsley, Manchester M28 3BP, UK e-mail: rakesh.mk@gmail.com 
Fig. 2 Tc-99m MDP bone scan
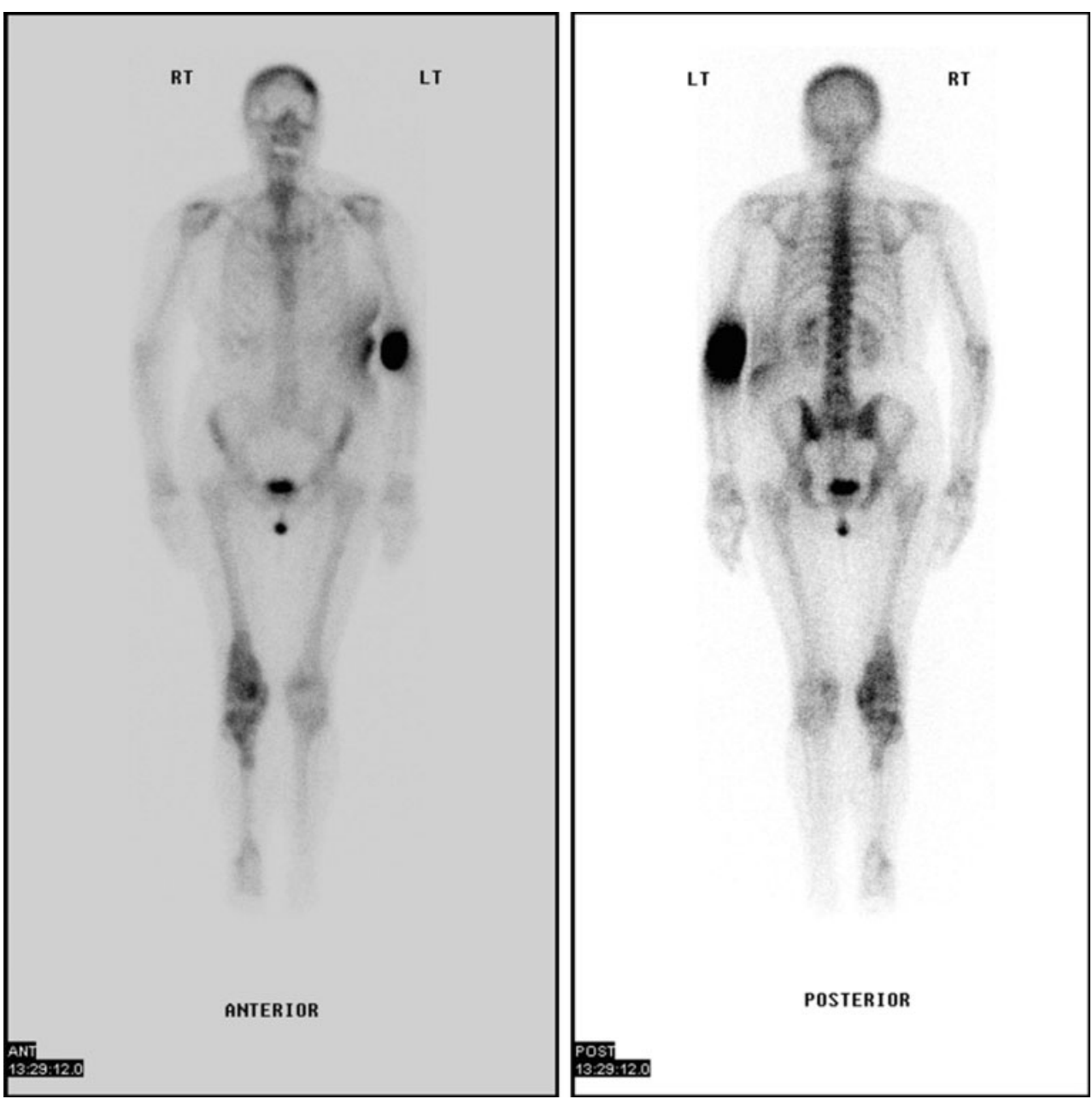
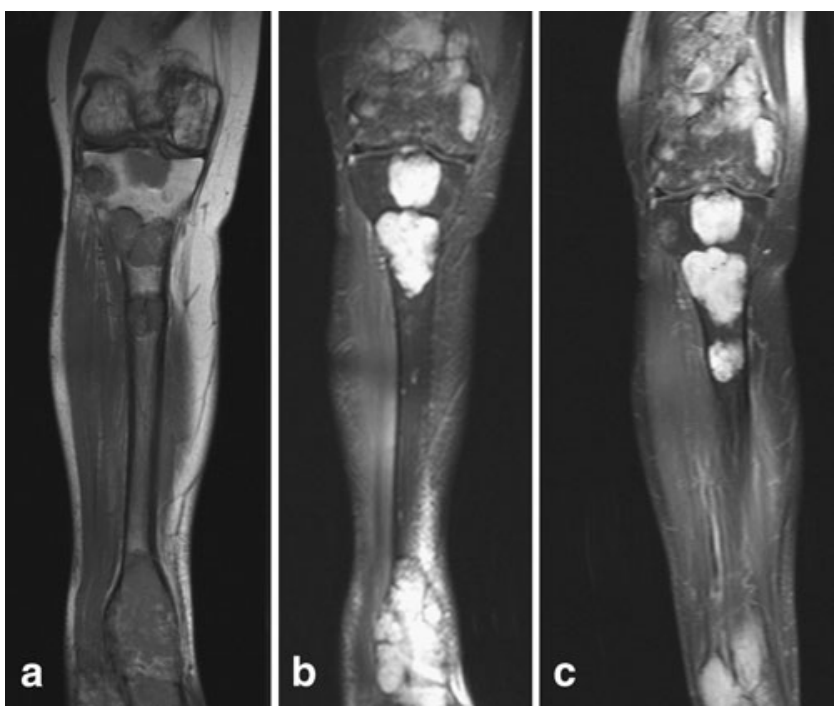

Fig. 3 Coronal MR images. a T1-weighted pre contrast. b STIR. c T1-weighted post contrast sequence

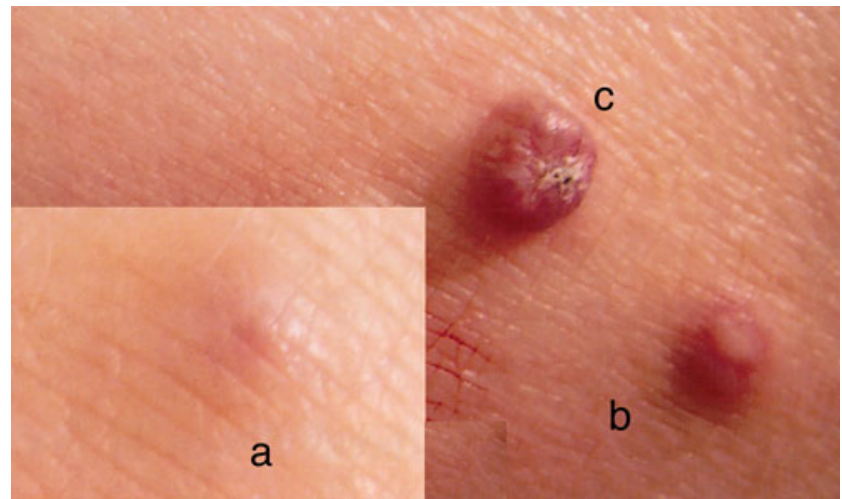

Fig. 4 Photographs of the skin lesions, in order of progression in time, from a to c 